\title{
Experimental Study on Peak Pressure of Shock Waves in Quasi-Shallow Water
}

\author{
Zhenxiong Wang, Wenbin Gu, and Jianqing Liu \\ College of Field Engineering, PLA University of Science and Technology, Nanjing, Jiangsu 210007, China \\ Correspondence should be addressed to Wenbin Gu; guwenbin1@aliyun.com
}

Received 2 November 2015; Accepted 10 December 2015

Academic Editor: Francesco Franco

Copyright (C) 2015 Zhenxiong Wang et al. This is an open access article distributed under the Creative Commons Attribution License, which permits unrestricted use, distribution, and reproduction in any medium, provided the original work is properly cited.

Based on the similarity laws of the explosion, this research develops similarity requirements of the small-scale experiments of underwater explosions and establishes a regression model for peak pressure of underwater shock waves under experimental condition. Small-scale experiments are carried out with two types of media at the bottom of the water and for different water depths. The peak pressure of underwater shock waves at different measuring points is acquired. A formula consistent with the similarity law of explosions is obtained and an analysis of the regression precision of the formula confirms its accuracy. Significance experiment indicates that the influence of distance between measuring points and charge on peak pressure of underwater shock wave is the greatest and that of water depth is the least within the range of geometric parameters. An analysis of data from experiments with different media at the bottom of the water reveals an influence on the peak pressure, as the peak pressure of a shock wave in a body of water with a bottom soft mud and rocks is about 1.33 times that of the case where the bottom material is only soft mud.

\section{Introduction}

Underwater shock wave generated by blasting charge in quasi-shallow water is an important reason causing damage to the target. And shock wave's damage is not only influenced by shape, structure, and material property of target buildings, but also affected by intensity of shock wave which is usually judged by its maximum pressure.

Development of experiment technology of underwater explosive load has brought much convenience to research on maximum pressure of underwater shock wave [1], and scholars at home and abroad have conducted numerous experiments and researched on the shock wave generated by the underwater explosion. With abundant experiments and predecessors' research production, Cole has comprehensively elaborated physical phenomenon and basic law of underwater explosion and established calculating formula on maximum pressure of explosion flow field, specific impulse, and energy density in certain range [2]. Slifko has carried out profound experiment research on pressure characteristics of explosion shock wave in the limitless water mediums, revealing that functional relationships exist among pressure of underwater shock wave, depth, and other factors [3]. Hanasaki and Ito have calculated maximum pressure of underwater pressure wave by measuring the largest particle velocity of water surface when using $3 \#$ detonator in underwater explosion [4], but it may not be practical in the actual measure for being influenced by duration and transmission distance of pressure wave. Takayama and Onodera have studied transmission of underwater spherical shock wave through micro explosion induced by shock of ruby laser to plumbum board, quantitatively describing movement and isopycnic line of underwater spherical shock wave [5]; Swisdak Jr. has analyzed the experimental results and discovered that charging radius and density affect the pressure of underwater shock wave [6]; Adushkin et al. have also carried out researches on shock wave and seismic wave of underwater explosion [7]; Ostapenko's analysis of stable, discontinuous, two-layer waves is of a purely theoretical nature, and the possibility of the existence of such waves requires a special experimental investigation [8]; through analyzing maximum pressure of underwater shock wave from four charging structures' underwater explosion, Zhou et al. have selected appropriate explosion for underwater arms 
TABLE 1: Relationship between similar items and coefficient scale.

\begin{tabular}{lc}
\hline Similar items & Coefficient scales \\
\hline Geometric similarity & Length coefficient scale $\lambda$ \\
Motion similarity & Velocity coefficient scale $\lambda / \tau$; acceleration coefficient scale $\lambda / \tau^{2}$ \\
Dynamic similarity & Pressure coefficient scale $\pi=\rho \varphi^{2}$; energy coefficient scale $\varepsilon=\pi \lambda^{2}$ \\
\hline
\end{tabular}

$\tau$ refers to time coefficient scale and $\rho$ is density of medium.

[9]; Kwang has reversed explosion of the Cheonan through analyzing its underwater shock wave [10]. In all, researches about underwater shock wave are mainly about experiments on maximum pressure of shock wave and pressure features of shock wave when it is under the influence of free surface as well as reversing some parameters of explosions through underwater shock wave.

In quasi-shallow water, shock wave from the explosion is influenced by water and bottom interfaces. And the shock wave goes through multiple reflections and transmissions, generating interactions with the wave system and the problem is even more complicated [11]. Main factors influencing maximum pressure are water depth, explosion height, and measuring points' position (horizontal distance from measuring point to charging center as well as distance from measuring point to water bottom); meanwhile, loading dose, performance parameter, and medium parameter of water bottom largely affect maximum pressure of shock wave. Due to complexity of water bottom factors, researches of pressure features of shock wave impacted by the water bottom are relatively few, and studies about pressure features of shock wave from the quasi-shallow water explosion impacted by both water surface and bottom are fewer [12]. Hence, on account of various factors' influence on shock wave, a reasonable theoretical calculating model is still extremely difficult to establish.

Building regression model of maximum pressure based on similarity theory, the present paper conducted the real explosion simulation experiments with $400 \mathrm{~g}$ press-fit TNT explosives. With different water bottom media, maximum pressure of shock wave from measuring points influenced by different factors is collected; then data fitting is adopted to regress calculation formula on maximum pressure of shock wave. Through significance experiment analysis, greater influential factors are displayed and calculating formula of the maximum pressure under different circumstances of the water bottom is demonstrated.

\section{Determination of Similar Requirements and Similar Conditions of Simulation Experiment}

To verify the similar law of underwater shock wave, Cole has conducted a large number of experiments to indicate that all parameters of underwater shock wave due to changes in dose and distance can be estimated accurately, and it proves that similarity law of the underwater explosion is the universal law of shock wave. But due to the special nature of near zone of charge, authentication area of similarity law is generally within the range of water pressure $180 \mathrm{MPa}$, equivalent to about seven times the area outside the radius of the spherical charge $[13,14]$. According to explosion similarity theory, at least three basic similar requirements between prototype experiment and simulation experiment exist, namely, geometric similarity, motion similarity, and dynamic similarity. The three basic requirements are necessary, but not sufficient. Relationship between similar items and corresponding coefficient scale is shown in Table 1.

From the viewpoint of energy similarity, in prototype experiment and simulation experiment, there exist the following relationships between those corresponding parameters in explosive field:

$$
\begin{aligned}
\text { Parameter: } P_{m}\left(t_{m}, X_{m}, Y_{m}, Z_{m}\right) & =P(t, X, Y, Z), \\
\text { Velocity: } u_{m}\left(t_{m}, X_{m}, Y_{m}, Z_{m}\right) & =u(t, X, Y, Z), \\
\text { Time: } t_{m} & =\left(\frac{W_{m}}{W}\right)^{1 / 3} t .
\end{aligned}
$$

2.1. Major Physical Parameters Restricting Explosion Parameters in Quasi-Shallow Water. Without considering water viscosity and gravity, parameters restricting explosion in quasishallow water include geometric parameters, water medium, parameters of water bottom medium, explosion parameters, and parameters of underwater shock wave. Restricting factors of shock wave in the quasi-shallow water explosion are in total 17, which are listed together with dimensions in Table 2.

2.2. Similarity Criterion. For 17 main physical parameters necessary to study, the research selects the 3 physical parameters $E\left(M L^{2} T^{-2}\right), r_{0}(L)$, and $C_{0}\left(L T^{-1}\right)$ to be the basic physical parameters. According to dimension's harmonious condition as well as $\pi$ theorem, 14 similarity criteria of independent dimensionless parameters can be listed in 3 categories:

Similarity criteria of the 1st category:

$$
\begin{aligned}
& \pi_{1}=\frac{d}{r_{0}} ; \\
& \pi_{2}=\frac{H}{r_{0}} ; \\
& \pi_{3}=\frac{L}{r_{0}} ; \\
& \pi_{4}=\frac{h}{r_{0}} ; \\
& \pi_{10}=\frac{t}{r_{0}} c_{0} .
\end{aligned}
$$


TABLE 2: 17 Major physical parameters restricting explosion.

\begin{tabular}{lclc}
\hline Number & Symbol & Name & Dimension \\
\hline 1 & $E_{0}$ & Total energy of charge & $M$ \\
2 & $r_{0}$ & Radius of charge & $M L^{2} T^{-2}$ \\
3 & $d$ & Depth of water & $L$ \\
4 & $H$ & Distance from charge to water bottom (explosion height) & $L$ \\
5 & $L$ & Horizontal distance from charging center to measuring point & $L$ \\
6 & $h$ & Distance from measuring point to water bottom (measuring height) \\
7 & $\rho_{0}$ & Initial density of water bottom medium & $M L^{-3}$ \\
8 & $\sigma$ & Compression strength of water bottom medium & $M L^{-1} T^{-2}$ \\
9 & $k_{0}$ & Cohesion of water bottom medium & $M L^{-1} T^{-2}$ \\
10 & $k_{1}$ & Modulus of plastic loading volume of water bottom medium & $M L^{-1} T^{-2}$ \\
11 & $p_{0}$ & Hydro-static pressure of water & $M L^{-1} T^{-2}$ \\
12 & $C_{0}$ & Acoustic velocity of water & $L T^{-1}$ \\
13 & $t$ & Time parameter & $T$ \\
14 & $p$ & Pressure of shock wave & $L T^{-1}$ \\
15 & $D$ & Velocity of shock wave & $M L^{-1} T^{-2}$ \\
16 & $u$ & Particle velocity rear shock wave & $L T^{-1}$ \\
$M L^{-3}$ & Particle intensity rear shock wave \\
\hline
\end{tabular}

Similarity criteria of the 2nd category:

$$
\begin{aligned}
& \pi_{5}=\frac{\rho_{0}}{E_{0}} r_{0}^{3} c_{0}^{2} ; \\
& \pi_{6}=\frac{\sigma}{E_{0}} r_{0}^{3} ; \\
& \pi_{7}=\frac{k_{0}}{E_{0}} r_{0}^{3} ; \\
& \pi_{8}=\frac{k_{1}}{E_{0}} r_{0}^{3} ; \\
& \pi_{9}=\frac{p_{0}}{E_{0}} r_{0}^{3} ; \\
& \pi_{11}=\frac{p}{E_{0}} r_{0}^{3} ; \\
& \pi_{14}=\frac{\rho}{E_{0}} r_{0}^{3} c_{0}^{2} .
\end{aligned}
$$

Similarity criteria of the 3rd category:

$$
\begin{aligned}
& \pi_{12}=\frac{D}{c_{0}} ; \\
& \pi_{13}=\frac{u}{c_{0}} .
\end{aligned}
$$

In line with basic theorem of dimension theory, any dimensionless characteristic parameter $\pi$ in and after explosion process can be above 14 independent dimensionless parameters:

$$
\pi=f\left(\pi_{1}, \pi_{2}, \ldots, \pi_{14}\right) .
$$

2.3. Analysis on Similarity Criterion. The above 14 dimensionless number groups constitute the model law. According to explosion similarity theory, the 14 dimensionless parameters between the model and the real should remain the same. Here, analysis on similarity criterion is shown with subscript " 1 " standing for the real and subscript " 2 " for the model:

By $\pi_{1}: d_{1} / r_{01}=d_{2} / r_{02} \Rightarrow d_{1} / d_{2}=r_{01} / r_{02}$; the depth of water is similar to charging radius;

By $\pi_{2}: H_{1} / r_{01}=H_{2} / r_{02} \Rightarrow H_{1} / H_{2}=r_{01} / r_{02}$; distance from charging center to water bottom is similar to charging radius;

By $\pi_{3}: L_{1} / r_{01}=L_{2} / r_{02} \Rightarrow L_{1} / L_{2}=r_{01} / r_{02}$; horizontal distance from charging to measuring point is similar to charging radius;

By $\pi_{4}: h_{1} / r_{01}=h_{2} / r_{02} \Rightarrow h_{1} / h_{2}=r_{01} / r_{02}$; distance from charging to water bottom is similar to charging radius;

Ву $\pi_{5}: \rho_{01} r_{01}^{3} C_{01}^{2} / E_{01}=\rho_{02} r_{02}^{3} C_{02}^{2} / E_{02}$; substituting with equation $E_{0}=C Q_{v}$, then $\rho_{01} r_{01}^{3} C_{01}^{2} / C_{1} Q_{v 1}=$ $\rho_{02} r_{02}^{3} C_{02}^{2} / C_{2} Q_{v 2}$; supposing $\rho_{01} Q_{v 2} C_{01}^{2} / \rho_{02} Q_{v 1} C_{02}^{1}=$ $r_{02}^{3} C_{1} / r_{01}^{3} C_{2}=1$, then water medium in experiment should be the same; $\rho_{01}=\rho_{02}, C_{01}=C_{02}$, and with the same explosive $Q_{v 1}=Q_{v 2}$;

By $\pi_{6}: \sigma_{1} r_{01}^{3} / E_{01}=\sigma_{2} r_{02}^{3} / E_{02} \Rightarrow \sigma_{1} r_{01}^{3} / C_{1} Q_{v 1}=$ $\sigma_{2} r_{02}^{3} / C_{2} Q_{v 2}$; if $C_{2} r_{01}^{3} / C_{1} r_{02}^{3}=1$, the same explosive as well as medium of water bottom is required;

Ву $\pi_{7}: k_{01} r_{01}^{3} / E_{01}=k_{02} r_{02}^{3} / E_{02} \Rightarrow k_{01} r_{01}^{3} / C_{1} Q_{v 1}=$ $k_{02} r_{02}^{3} / C_{2} Q_{v 2}$; provided $C_{1} r_{01}^{3} / C_{2} r_{02}^{3}=1$, thus the same explosive and medium of water bottom should be realized;

Ву $\pi_{8}: k_{1} r_{01}^{3} / E_{01}=k_{2} r_{02}^{3} / E_{02} \Rightarrow k_{1} r_{01}^{3} / C_{1} Q_{v 1}=$ $k_{2} r_{02}^{3} / C_{2} Q_{v 2}$; provided $C_{1} r_{01}^{3} / C_{2} r_{02}^{3}=1$, then the 
same explosive and medium of water bottom are required;

By $\pi_{9}: P_{01} r_{01}^{3} / E_{01}=P_{02} r_{02}^{3} / E_{02} \Rightarrow P_{01} r_{01}^{3} / C_{1} Q_{v 1}=$ $P_{02} r_{02}^{3} / C_{2} Q_{v 2}$; supposing $C_{1} r_{01}^{3} / C_{2} r_{02}^{3}=1$, the same explosive as well as hydrostatic pressure of water bottom is required;

By $\pi_{10}: t_{1} r_{01}^{3} / E_{01}=t_{2} r_{02}^{3} / E_{02} \Rightarrow t_{1} / t_{2}=r_{01} C_{02} /$ $r_{02} C_{01}$; if water media are the same $\left(C_{01}=C_{02}\right)$, time parameter is geometrically similar to charging radius; By $\pi_{11}: P_{m 1} r_{01}^{3} / E_{01}=P_{m 2} r_{02}^{3} / E_{02} \Rightarrow P_{m 1} r_{01}^{3} / C_{1} Q_{v 1}=$ $P_{m 2} r_{02}^{3} / C_{2} Q_{v 2}$; if $C_{1} r_{01}^{3} / C_{2} r_{02}^{3}=1$, with $P_{m 1}=P_{m 2}$ required, the same explosive should be adopted;

By $\pi_{12}: D_{1} / C_{01}=D_{2} / C_{02}$, with $D_{1}=D_{2}$ required; then water media in experiment should be the same;

By $\pi_{13}: u_{1} / C_{01}=u_{2} / C_{02}$, and, with $u_{1}=u_{2}$ required, water media in experiment should be the same;

By $\pi_{14}: \rho_{1} r_{01}^{3} C_{01}^{2} / E_{01}=\rho_{2} r_{02}^{3} C_{02}^{2} / E_{02} \Rightarrow \rho_{1} r_{01}^{3} C_{01}^{2} /$ $C_{1} Q_{v 1}=\rho_{2} r_{02}^{3} C_{02}^{2} / C_{2} Q_{v 2}$, on condition that $C_{1} r_{01}^{3} /$ $C_{2} r_{02}^{3}=1$, and with $\rho_{1}=\rho_{2}$ required; therefore, the same explosive and medium of water bottom should be realized.

From the above analysis, similarity conditions of simulation experiment can be concluded as

(1) employing the same water medium in experiment (including equal hydrostatic pressure) and the same geological conditions,

(2) employing explosives of the same type and density,

(3) depth of water which is similar to distance from charging to water bottom; horizontal distance from charging center to measuring point is similar to charging radius and distance from measuring point to water bottom is geometrically similar to charging radius,

(4) charging shapes which are geometrically similar and charging dose is directly proportional to the cube of charging radius.

If the above similarity conditions for simulation experiment are tenable, that is, the prototype experiment is physically similar to simulation experiment, the experiment results can be possible to achieve and meaningful parameters are the four similarity criteria of the 1st category; then

$$
\pi=f\left(\frac{d}{r_{0}}, \frac{H}{r_{0}}, \frac{L}{r_{0}}, \frac{t}{r_{0}} c_{0}\right) .
$$

\section{Establishment of Regression Model of Maximum Pressure and Regression Analysis Method}

3.1. Fitted Model of Maximum Pressure. On basis of satisfying similar conditions, assuming that group charge explodes in quasi-shallow water, static pressure and density of water are $p_{0}$ and $\rho_{0}$. The overpressure of spatial point $\Delta p_{m}=p_{m}-p_{0}$

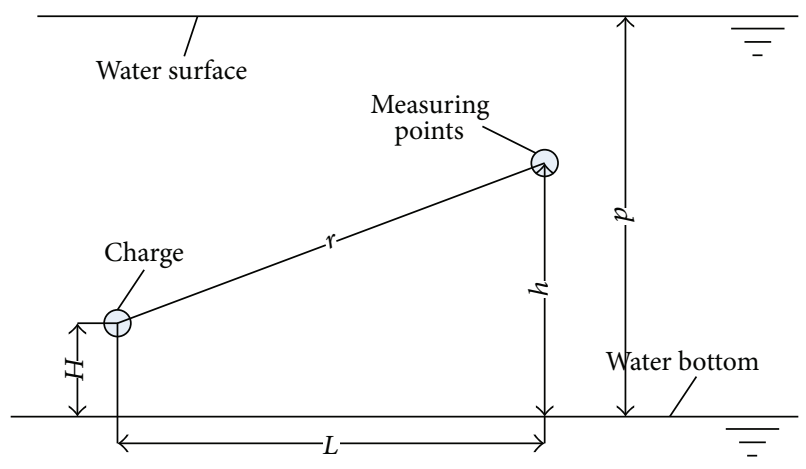

FIGURE 1: Relative location diagram of measuring points.

( $p_{m}$ is maximum pressure of incident wave surface; as $p_{m} \gg$ $p_{0}, \Delta p_{m} \approx p_{i}$ ) is determined by total energy of charging $E_{0}$, distance from charging to water bottom $H$, distance from measuring point to water bottom $h$, depth of water $d$, and horizontal distance from measuring point to charging $L$ as well as $p_{0}$ and $\rho_{0}$, with relative location diagram shown in Figure 1 .

And its function expression is

$$
\Delta p_{m}=f\left(H, h, d, L, E_{0}, p_{0}, \rho_{0}\right) .
$$

That is to say,

$$
p_{m}=f\left(H, h, d, L, E_{0}, p_{0}, \rho_{0}\right) .
$$

If $E, p_{0}$, and $\rho_{0}$ are selected as independent variables, according to $\pi$ theorem:

$$
\begin{aligned}
& \pi_{1}=\frac{p_{m}}{p_{0}} \\
& \pi_{2}=\frac{H p_{0}^{1 / 3}}{E_{0}^{1 / 3}} \\
& \pi_{3}=\frac{h p_{0}^{1 / 3}}{E_{0}^{1 / 3}} \\
& \pi_{4}=\frac{d p_{0}^{1 / 3}}{E_{0}^{1 / 3}} \\
& \pi_{5}=\frac{L p_{0}^{1 / 3}}{E_{0}^{1 / 3}} .
\end{aligned}
$$

Hence, relationship among maximum pressure, charging, and measuring points can be demonstrated as

$$
F\left(\frac{p_{m}}{p_{0}}, \frac{H p_{0}^{1 / 3}}{E_{0}^{1 / 3}}, \frac{h p_{0}^{1 / 3}}{E_{0}^{1 / 3}} \frac{d p_{0}^{1 / 3}}{E_{0}^{1 / 3}}, \frac{L p_{0}^{1 / 3}}{E_{0}^{1 / 3}}\right)=0 .
$$

As $E=C Q_{v}$ (C refers to mass of charging and $Q_{v}$ explosion heat), when $Q_{v}$ and $p_{0}$ are determined, the above equation can be transformed into

$$
p_{m}=F_{1}\left(\frac{H}{\sqrt[3]{C}}, \frac{h}{\sqrt[3]{C}}, \frac{d}{\sqrt[3]{C}}, \frac{L}{\sqrt[3]{C}}\right) .
$$


If $\bar{H}=H / \sqrt[3]{C}, \bar{h}=h / \sqrt[3]{C}, \bar{d}=d / \sqrt[3]{C}$, and $\bar{L}=L / \sqrt[3]{C}$ are, respectively, scale shocking height, scale measurement height, scale depth of water, and scale horizontal distance, then $F_{1}$ can be

$$
F_{1}(\bar{H}, \bar{h}, \bar{d}, \bar{L})=A \bar{H}^{b_{1}} \bar{h}^{b_{2}} \bar{d}^{b_{3}} \bar{L}^{b_{4}}
$$

Then,

$$
p_{m}=A \bar{H}^{b_{1}} \bar{h}^{b_{2}} \bar{d}^{b_{3}} \bar{L}^{b_{4}} .
$$

In the equation, coefficient $A$ and indexes $b_{1}, b_{2}, b_{3}$, and $b_{4}$ are determined by the experiment.

3.2. Regression Method of Experimental Coefficient. Take logarithm of both sides of (13) and nonlinear equation turns into linear equation:

$$
\ln p_{m}=\ln A+b_{1} \ln \bar{H}+b_{2} \ln \bar{h}+b_{3} \ln \bar{d}+b_{4} \ln \bar{L}
$$

When $X_{1}=\ln \bar{H}, X_{2}=\ln \bar{h}, X_{3}=\ln \bar{d}, X_{4}=\ln \bar{L}, X_{5}=Y=$ $\ln p_{m}$, and $b_{0}=\ln A$, then (14) is

$$
Y=b_{0}+b_{1} X_{1}+b_{2} X_{2}+b_{3} X_{3}+b_{4} X_{4} \text {. }
$$

According to least square method, select $b_{0}, b_{1}, b_{2}, b_{3}$, and $b_{4}$ to get residual sum of square:

$$
\begin{aligned}
& \text { Q } \\
& =\sum_{j=1}^{n}\left(Y_{j}-\left(b_{0}+b_{1} X_{1 j}+b_{2} X_{2 j}+b_{3} X_{3 j}+b_{4} X_{4 j}\right)\right)^{2} .
\end{aligned}
$$

Here, $n$ is the number of measuring points in maximum pressure statistics; $Y_{j}$ is the $j$ th maximum pressure; $X_{1 j}$, $X_{2 j}, X_{3 j}$, and $X_{4 j}$ are, respectively, set parameters of $Y_{j}$ corresponding to variables $X_{1}, X_{2}, X_{3}$, and $X_{4}$ in (15). If $Q$ has a minimum value, $b_{0}, b_{1}, b_{2}, b_{3}$, and $b_{4}$ need to meet

$$
\frac{\partial Q}{\partial b_{i}}=0 \quad(i=0,1, \ldots, 4) \text {. }
$$

Equation set can be induced from (17):

$$
\begin{aligned}
& l_{11} b_{1}+l_{12} b_{2}+l_{13} b_{3}+l_{14} b_{4}=l_{15}, \\
& l_{21} b_{1}+l_{22} b_{2}+l_{23} b_{3}+l_{24} b_{4}=l_{25}, \\
& l_{31} b_{1}+l_{32} b_{2}+l_{33} b_{3}+l_{34} b_{4}=l_{35}, \\
& l_{41} b_{1}+l_{42} b_{2}+l_{43} b_{3}+l_{44} b_{4}=l_{45} .
\end{aligned}
$$

In the equation set,

$$
l_{i j}=l_{j i}=\sum X_{i} X_{j}-\frac{1}{n}\left(\sum X_{i}\right)\left(\sum X_{j}\right) .
$$

And solving coefficient matrix of equation set (18), $b_{0}, b_{1}, b_{2}$, $b_{3}$, and $b_{4}$ can be obtained.

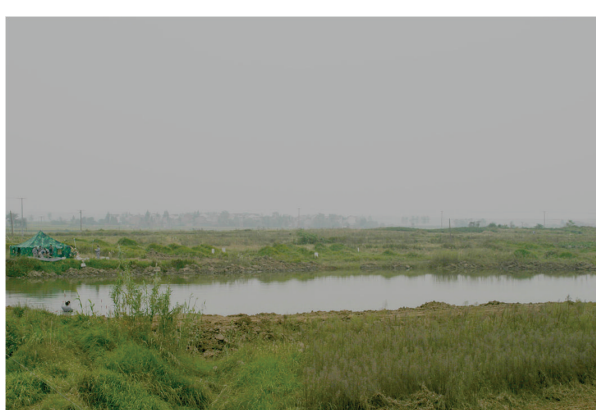

Figure 2: Experiment area.

3.3. Analysis on Regression Accuracy. In multiple linear regressions, correlative coefficients between variables are complex for correlation may exist between any two variables. When independent variables are highly correlated, it may be really difficult for factor analysis. Correlation coefficient between $X_{i}$ and $X_{j}$ can be obtained by the following equation:

$$
r_{i j}=\sqrt{\frac{\sum_{i=0}^{4}\left(\sum_{j=1}^{n}\left(X_{i j}-\overline{X_{i}}\right)\left(Y_{j}-\bar{Y}\right) b_{i}\right)}{\sum_{j=1}^{n}\left(Y_{j}-\bar{Y}\right)^{2}}} .
$$

Here, $\overline{X_{i}}=(1 / n) \sum_{j=1}^{n} X_{i j}(i=0,1, \ldots, 4)$.

When the regression coefficient is obtained, regression accuracy should also be calculated and analyzed, including the residual sums of square:

$$
U=\sum(\widehat{Y}-\bar{Y})^{2}=\sum_{i=0}^{k} b_{i} l_{i k} \quad(k=0,1, \ldots, 4) \text {. }
$$

The bigger it is, the stronger the regression regularity is, and so are the effects. And the residual standard deviation is

$$
s=\sqrt{\frac{Q}{n-k-1}} .
$$

Regression mean square to residual mean square ratio is

$$
F=\frac{U / k}{Q /(n-k-1)}=\frac{U}{k s^{2}} .
$$

Significance experiment can be conducted on the whole regression with value of $F$, and it would analyze whether linear relationship between $Y$ and $k$ independent variables $X_{1}, X_{2}, X_{3}, \ldots, X_{k}$ is significant.

\section{Simulation and Analysis on Experiment Data}

To study influential factors of peak pressure of underwater shock wave, three-dimensional press shot of $400 \mathrm{~g}$ is employed in simulation experiment, and settings of blast experiment area and sensor are shown in Figures 2 and 3. Through measuring peak pressure of measuring points with different water depths, explosion height, measuring height, 
TABLE 3: Statistics of incident pressure at part of the measuring points in slime water bottom.

\begin{tabular}{lccccccccccc}
$\overline{\bar{H}}$ & $\bar{h}$ & $\bar{d}$ & $\bar{L}$ & $p_{m}$ & $p_{\infty}$ & $\bar{H}$ & $\bar{h}$ & $\bar{d}$ & $\bar{L}$ & $p_{m}$ & $p_{\infty}$ \\
\hline 0.05 & 0.01 & 0.81 & 1.45 & 18.0 & 34.4 & 0.14 & 0.26 & 0.96 & 1.78 & 16.6 & 27.2 \\
0.05 & 0.01 & 0.84 & 1.94 & 13.3 & 24.7 & 0.14 & 0.65 & 0.81 & 1.45 & 21.5 & 32.2 \\
0.05 & 0.26 & 0.84 & 1.45 & 18.5 & 34.0 & 0.14 & 0.65 & 0.84 & 1.45 & 20.3 & 32.2 \\
0.05 & 0.26 & 0.96 & 1.62 & 16.4 & 30.0 & 0.14 & 0.65 & 0.96 & 1.62 & 18.7 & 28.7 \\
0.05 & 0.52 & 0.81 & 1.94 & 14.5 & 23.9 & 0.26 & 0.01 & 0.81 & 1.62 & 18.0 & 29.9 \\
0.05 & 0.65 & 0.84 & 1.45 & 20.7 & 31.4 & 0.26 & 0.01 & 0.84 & 1.45 & 25.0 & 33.8 \\
0.05 & 0.65 & 0.96 & 1.62 & 18.8 & 28.2 & 0.26 & 0.26 & 0.84 & 1.45 & 20.3 & 34.4 \\
0.14 & 0.01 & 0.84 & 1.45 & 20.7 & 34.2 & 0.26 & 0.26 & 0.96 & 1.62 & 22.3 & 30.3 \\
0.14 & 0.01 & 0.96 & 2.10 & 14.1 & 22.6 & 0.26 & 0.65 & 0.81 & 1.45 & 24.6 & 33.0 \\
0.14 & 0.26 & 0.84 & 1.45 & 21.9 & 34.2 & 0.26 & 0.65 & 0.96 & 1.94 & 19.5 & 24.2 \\
\hline
\end{tabular}

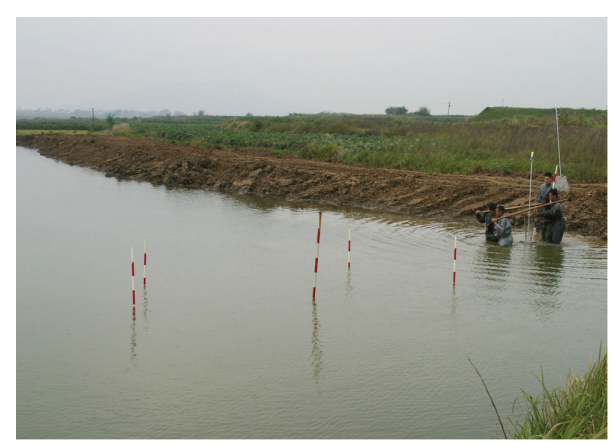

FIGURE 3: Layout area of charging blast and sensor.

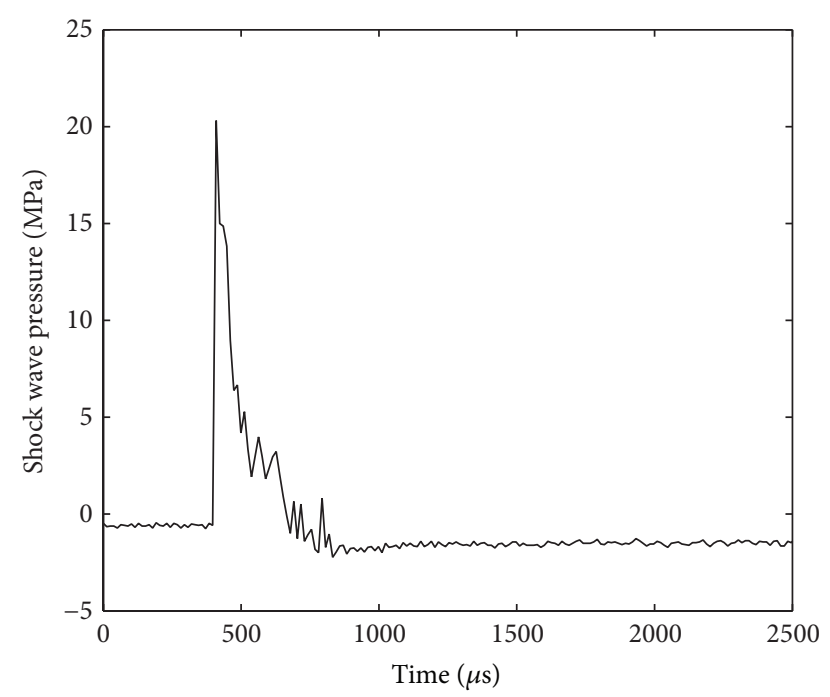

FIgURE 4: Curve of typical flush water shock wave collected by sensor.

and horizontal distance and monitoring measuring points in different underwater medium, peak pressure under different circumstances can be obtained. Export the measured data and process it with MATLAB; then history curve of shock wave pressure is attained and history curve of typical shock wave pressure at measuring points is shown in Figure 4. The data are processed according to regression model of
Section 3; afterwards main factors influencing peak pressure at measuring points of the explosion in quasi-shallow water are analyzed. Formulas on peak pressure in quasi-shallow water are obtained through fitting, and then regression accuracy from the equation is analyzed and verified.

\subsection{Research on Peak Pressure of Shock Wave of Slime Water Bottom}

4.1.1. Measuring Data of Underwater Shock Wave Pressure of Slime Water Bottom. Figure 4 shows that, in simulation experiment, MATLAB helps obtain the history curve of typical shock wave pressure at measuring points in water [15], which shows that the change of shock wave pressure is more complex than attenuation of shock wave pressure in the unlimited water explosion. Due to impact of water bottom and surface, there appears obvious attenuation of the shock wave at measuring points, influenced by change of measuring sites. There are many factors influencing shock wave [16], and the present paper mainly studies the influence of water depth, measuring depth, distance, and so on upon peak pressure of shock wave; hence, factors affecting wave change at measuring points would not be analyzed in detail, and peak pressure would be collected and processed.

Under the circumstance of slime water bottom, experiment results of peak pressure of part of measuring points in explosion field of simulation experiment are obtained, as is shown in Table 3 , where $p_{\infty}$ refers to peak pressure of shock wave at underwater measuring points when charge explodes in unlimited water medium with rational distance $r$ calculated by Cole formula [14]:

$$
p_{\infty}=52.3 \bar{r}^{-1.13}\left(10^{6} \mathrm{~Pa}\right) \quad(0.637<\bar{r}<12.821) .
$$

In Table 3, $p_{m}$ is measured pressure of incident wave front of explosion in quasi-shallow water; $p_{\infty}$ is pressure of incident wave front at a distance in the same proportion in infinite water medium with unit of MPa.

4.1.2. Data Fitting and Regression Analysis. By least square method, data in Table 1 are analyzed by regression as 
TABLe 4: $F$ test.

\begin{tabular}{ccccc}
\hline & $X_{1}$ & $X_{2}$ & $X_{3}$ & $X_{4}$ \\
\hline$p_{i}$ & 0.20070 & 0.0862 & 0.0004 & 0.2484 \\
$F_{i}$ & 30.5011 & 13.1025 & 0.0631 & 37.7578 \\
\hline
\end{tabular}

$b_{0}=3.83, b_{1}=0.1507, b_{2}=0.0243, b_{3}=0.0403$, and $b_{4}=-1.054$. And regression equation is

$$
\begin{aligned}
\widehat{Y}= & 3.83+0.151 X_{1}+0.024 X_{2}+0.040 X_{3} \\
& -1.054 X_{4} .
\end{aligned}
$$

Regression variables $X_{1}, X_{2}, X_{3}$, and $X_{4}$ arrange importance of peak pressure's expected value $\hat{Y}$, as is in Table 2 . Then significance experiment ( $F$ test) is carried out. In the table, $p_{i}=b_{i}^{2} / c_{i i}, c_{i i}$ is the value on diagonal in inverse matrix of coefficient matrix in (18), and $F_{i}=p_{i} / s^{2}$.

Table 4 shows that $X_{4}$ affects $Y$ most meaningfully, $X_{1}$ comes second, and $X_{3}$ does not importantly impact $Y$. Therefore, the influence of horizontal distance from charging center to measuring point on peak pressure of incident front wave is the most consequential, explosion height comes second, and then comes measuring height; water depth does not have important influence.

From the viewpoint of energy distribution, since the equivalent charging radius of $400 \mathrm{~g}$, TNT Charging is $r_{0}=$ $4 \mathrm{~cm}$; water depth $H=47 \mathrm{~cm}$. Clearly, $H>7 r_{0}$, so energy generated by the explosion is mainly used for forming shock wave and underwater pits of explosion and the energy does not overflow water or form air shock wave. And it is consistent with regression conclusion "water depth does not significantly influence peak pressure of incident." Hence, in explosion experiment of quasi-shallow water, water depth can be ignored in researching peak value of measuring points within a flow field [1]. After removing items including $X_{3}$ in (25), experiment data are reanalyzed by regression as $b_{0}=$ $3.8225, b_{1}=0.1515, b_{2}=0.0246$, and $b_{4}=-1.0462$. Thus, regression equation of the experiment results is

$$
\widehat{Y}=3.823+0.152 X_{1}+0.025 X_{2}-1.046 X_{4} .
$$

Calculating results of simple correlation coefficients among regression variables are in Table 5 . As can be seen from the table, variables have little correlation between each other. Replace $\widehat{Y}\left(X_{5}\right)$ in formula (26) with $\ln p_{m}$ and $X_{1}, X_{2}$, and $X_{4}$ with $\ln \bar{H}, \ln \bar{h}$, and $\ln \bar{L}$, respectively; then, in experiment of soft mud, when single charge explodes in quasi-shallow water, calculating formula of shock wave pressure of measuring points in flow field is

$$
p_{m}=45.6 \bar{H}^{0.152} \bar{h}^{-0.025} \bar{L}^{-1.046} \text {. }
$$

Fitting range of (27) is $0.0529 \leq \bar{H} \leq 0.258 ; 0.0068 \leq \bar{h} \leq$ $0.244 ; 0.814 \leq \bar{d} \leq 0.964 ; 1.45 \leq \bar{L} \leq 2.10$.

Calculate peak pressure of some measuring points with (27) peak pressure shock wave equation (27) and then compare results with actually measured peak pressure and the
TABLE 5: Calculation of simple correlation coefficients among regression variables.

\begin{tabular}{lcccc}
\hline$r_{i j}$ & $X_{1}$ & $X_{2}$ & $X_{4}$ & $X_{5}=Y$ \\
\hline$X_{1}$ & 1.0000 & 0.0013 & -0.0803 & 0.5430 \\
$X_{2}$ & 0.0013 & 1.0000 & -0.0000 & 0.2276 \\
$X_{4}$ & -0.0803 & -0.0000 & 1.0000 & -0.7617 \\
$X_{5}=Y$ & 0.5430 & 0.2276 & -0.7617 & 1.0000 \\
\hline
\end{tabular}

details are shown in Table 6, where the unit of measured and calculated $p_{m}$ is MPa.

Analysis in Table 6 shows that relative error between calculated peak pressure at 33 measuring points and corresponding actual data is between $0.49 \%$ and $15.62 \%$, and there is only one point where the relative error is beyond $15 \%$, and it should be regarded as singular experiment data. Therefore, singular results in measurement can be eliminated through comparing back-stepped peak pressure calculated by regression formula with actually measured pressure and analyzing experiment wave form. There are seven measuring points where the relative error is between $5 \%$ and $10 \%$, accounting for $20 \%$, and those of the remaining points are smaller than 5\%. In a word, regression formula can be employed in calculating peak pressure of the shock wave of the single charge explosion in the quasi-shallow water flow field. And accuracy of the calculating results is relatively high in formula scope.

\subsection{Studies on Peak Pressure of Shock Wave in Water Bottom of Soft Mud with Rocks}

4.2.1. Peak Pressure of Shock Wave in Water Bottom of Soft Mud with Rocks. Under the condition at the water bottom of soft mud with rocks, in model experiment of actual explosion, actual results of experimented peak pressure of some measuring points within the explosion flow field are listed in Table 7.

4.2.2. Data Fitting and Regression Analysis. The least square method can be adopted to calculate data in Table 7 on regression. Through $F$ Experiment and analysis, it is shown that water depth $X_{3}$ does not significantly influence pressure regression parameter $X_{5}$. Thus, under the experiment condition, water depth could be ignored in regression of peak pressure of the shock wave. Experiment data of regression are $b_{0}=3.83 \mathrm{MPa}, b_{1}=0.097, b_{2}=0.017$, and $b_{4}=-0.903$. Regression equation is

$$
\widehat{Y}=3.83+0.097 X_{1}+0.017 X_{2}-0.903 X_{4} .
$$

Therefore, under the condition at the water bottom of soft mud with rocks, in the explosion of single charge in quasishallow water, calculating formula of peak pressure of the shock wave at measuring points within the flow field is

$$
p_{m}=48.4 \bar{H}^{0.097} \bar{h}^{0.017} \bar{L}^{-0.903} \text {. }
$$

In the equation, fitting range is $0.0529 \leq \bar{H} \leq 0.258 ; 0.0068 \leq$ $\bar{h} \leq 0.244 ; 0.814 \leq \bar{d} \leq 0.964 ; 1.45 \leq \bar{L} \leq 2.10$. 
TABLE 6: Comparisons of calculating results by regression and measured results.

\begin{tabular}{lcccccccccccc}
\hline $\bar{H}$ & $\bar{h}$ & $\bar{L}$ & $A-p_{m}$ & $C-p_{m}$ & $R$ & $\bar{H}$ & $\bar{h}$ & $\bar{L}$ & $A-p_{m}$ & $C-p_{m}$ & $R$ \\
\hline 0.05 & 0.0068 & 1.45 & 17.91 & 17.42 & 2.74 & 0.14 & 0.65 & 1.45 & 22.48 & 22.59 & 0.49 \\
0.05 & 0.0068 & 1.94 & 12.34 & 12.95 & 4.94 & 0.14 & 0.65 & 1.62 & 19.48 & 20.27 & 4.06 \\
0.05 & 0.26 & 1.45 & 18.51 & 19.11 & 3.24 & 0.14 & 0.65 & 1.94 & 17.90 & 16.8 & 6.15 \\
0.05 & 0.26 & 1.62 & 16.45 & 17.14 & 4.20 & 0.14 & 0.65 & 2.10 & 16.40 & 15.47 & 5.67 \\
0.05 & 0.26 & 1.78 & 16.55 & 15.54 & 6.10 & 0.26 & 0.0068 & 1.45 & 21.95 & 22.18 & 1.05 \\
0.05 & 0.26 & 2.10 & 14.44 & 13.08 & 9.42 & 0.26 & 0.0068 & 1.62 & 18.07 & 19.90 & 10.13 \\
0.05 & 0.52 & 1.94 & 14.63 & 14.46 & 1.16 & 0.26 & 0.0068 & 1.78 & 19.47 & 18.04 & 7.34 \\
0.05 & 0.52 & 2.10 & 12.63 & 13.32 & 5.46 & 0.26 & 0.0068 & 2.10 & 15.2 & 15.19 & 0.66 \\
0.05 & 0.65 & 1.45 & 20.20 & 19.57 & 3.12 & 0.26 & 0.26 & 1.45 & 23.88 & 24.34 \\
0.05 & 0.65 & 1.62 & 18.05 & 17.55 & 2.77 & 0.26 & 0.26 & 1.62 & 22.33 & 21.83 & 2.24 \\
0.05 & 0.65 & 2.10 & 13.09 & 13.40 & 2.37 & 0.26 & 0.26 & 1.78 & 21.02 & 19.79 & 5.85 \\
0.14 & 0.0068 & 1.45 & 20.86 & 20.11 & 3.60 & 0.26 & 0.26 & 1.94 & 18.52 & 18.09 \\
0.14 & 0.0068 & 1.94 & 12.93 & 14.95 & 15.62 & 0.26 & 0.65 & 1.45 & 25.63 & 24.92 & 2.32 \\
0.14 & 0.0068 & 2.10 & 14.13 & 13.77 & 5.48 & 0.26 & 0.65 & 1.62 & 22.77 & 22.35 & 1.84 \\
0.14 & 0.26 & 1.45 & 21.98 & 22.06 & 0.36 & 0.26 & 0.65 & 1.94 & 19.55 & 18.53 & 5.22 \\
0.14 & 0.26 & 1.78 & 18.72 & 17.94 & 4.17 & 0.26 & 0.65 & 2.10 & 15.59 & 17.06 & 9.43 \\
0.14 & 0.26 & 1.94 & 16.61 & 16.40 & 1.26 & & & & \\
\hline$A$
\end{tabular}

$A-p_{m}:$ actually measured $p_{m} ; C-p_{m}:$ calculated $p_{m} ; R$ : relative error (\%).

TABLE 7: Incident wave pressure of some measuring points under the condition of water bottom of soft mud with rocks.

\begin{tabular}{lcccccccccccc}
$\overline{\bar{H}}$ & $\bar{h}$ & $\bar{d}$ & $\bar{L}$ & $p_{m}$ & $p_{\infty}$ & $\bar{H}$ & $\bar{h}$ & $\bar{d}$ & $\bar{L}$ & $p_{m}$ & $p_{\infty}$ \\
\hline 0.05 & 0.01 & 0.81 & 1.45 & 26.6 & 34.4 & 0.14 & 0.65 & 0.84 & 1.45 & 25.8 & 32.2 \\
0.05 & 0.01 & 0.81 & 1.62 & 21.1 & 30.3 & 0.14 & 0.65 & 0.81 & 1.62 & 25.0 & 28.7 \\
0.05 & 0.01 & 0.81 & 1.78 & 18.8 & 27.3 & 0.14 & 0.65 & 0.81 & 1.78 & 22.3 & 26.1 \\
0.05 & 0.01 & 0.96 & 2.10 & 15.2 & 22.6 & 0.14 & 0.65 & 0.81 & 1.94 & 22.7 & 23.8 \\
0.05 & 0.26 & 0.84 & 1.45 & 24.8 & 34.0 & 0.14 & 0.65 & 0.81 & 2.10 & 20.3 & 21.9 \\
0.05 & 0.26 & 0.96 & 1.62 & 22.7 & 30.0 & 0.26 & 0.01 & 0.81 & 1.45 & 28.9 & 33.8 \\
0.05 & 0.26 & 0.96 & 1.78 & 21.7 & 27.0 & 0.26 & 0.01 & 0.81 & 1.94 & 20.3 \\
0.05 & 0.26 & 0.96 & 1.94 & 20.6 & 24.6 & 0.26 & 0.01 & 0.96 & 1.62 & 28.9 & 29.9 \\
0.05 & 0.26 & 0.81 & 2.10 & 20.3 & 22.5 & 0.26 & 0.26 & 0.84 & 1.45 & 29.7 & 34.4 \\
0.05 & 0.65 & 0.81 & 1.94 & 23.8 & 23.9 & 0.26 & 0.26 & 0.96 & 1.62 & 26.6 & 30.3 \\
0.05 & 0.65 & 0.96 & 1.78 & 23.1 & 25.7 & 0.26 & 0.65 & 0.81 & 1.94 & 21.5 & 24.2 \\
0.14 & 0.01 & 0.81 & 1.45 & 24.4 & 34.2 & 0.26 & 0.65 & 0.96 & 1.62 & 27.3 & 29.4 \\
0.14 & 0.01 & 0.81 & 1.62 & 21.4 & 30.2 & 0.26 & 0.65 & 0.96 & 1.78 & 24.6 \\
0.14 & 0.26 & 0.81 & 2.10 & 19.5 & 22.6 & 0.26 & 0.65 & 0.96 & 2.10 & 19.9 \\
\hline
\end{tabular}

When (29) is employed in calculating explosion in the water bottom of mud with rocks or in quasi-shallow water, actually measured peak pressure and peak pressure of the shock wave at some measuring points are shown in Table 8.

Comparison on measured value and calculated value from the regression formula at the same measuring points displays that comparative error is between $0.06 \%$ and $10.07 \%$. There are 32 measuring points with proportional error smaller than $5 \%$ and only one point larger than $10 \%$. Consequently, regression formula can be employed in calculating peak pressure of the shock wave of a single charge explosion in the quasi-shallow water flow field. And accuracy of the calculating results is relatively high in formula scope.
4.3. Analysis on Influence of Water Bottom Medium on Peak Pressure of Shock Wave. Figure 5 demonstrates change curve where theoretical and experimental values change with proportional distance. With the water bottom of mud with rocks, peak pressure of measuring points distributes near the theoretical values. Comparison of two pictures shows that peak pressure at measuring points on the water bottom of mud with rocks is higher than that of the water bottom of soft mud, which is caused by reflective wave to the water bottom and surface cut off pressure of shock wave and different interactions. To study the influence of different media of the water bottom on peak pressure, experiments are carried out in two different media of water bottom and peak pressure at the same measuring points which are shown in Table 9. 
TABLE 8: Actually measured peak pressure and calculated peak pressure of shock wave in water bottom of mud with rocks.

\begin{tabular}{|c|c|c|c|c|c|c|c|c|c|c|c|}
\hline $\bar{H}$ & $\bar{h}$ & $\bar{L}$ & $A-p_{m}$ & $C-p_{m}$ & $R$ & $\bar{H}$ & $\bar{h}$ & $\bar{L}$ & $A-p_{m}$ & $C-p_{m}$ & $R$ \\
\hline 0.05 & 0.0068 & 1.45 & 26.61 & 23.93 & 10.07 & 0.14 & 0.0068 & 2.10 & 17.08 & 18.24 & 6.79 \\
\hline 0.05 & 0.0068 & 1.62 & 21.12 & 21.55 & 2.00 & 0.14 & 0.26 & 1.62 & 26.08 & 25.28 & 3.07 \\
\hline 0.05 & 0.0068 & 1.78 & 19.04 & 19.61 & 2.99 & 0.14 & 0.26 & 1.62 & 20.85 & 21.10 & 1.20 \\
\hline 0.05 & 0.0068 & 1.94 & 17.98 & 17.99 & 0.06 & 0.14 & 0.26 & 2.10 & 19.48 & 19.49 & 0.05 \\
\hline 0.05 & 0.26 & 1.45 & 24.90 & 25.57 & 2.69 & 0.14 & 0.65 & 1.62 & 25.04 & 25.71 & 2.67 \\
\hline 0.05 & 0.26 & 1.62 & 22.69 & 23.04 & 1.54 & 0.14 & 0.65 & 1.78 & 22.30 & 23.39 & 4.89 \\
\hline 0.05 & 0.26 & 1.78 & 21.78 & 20.96 & 3.76 & 0.14 & 0.65 & 1.94 & 22.27 & 21.46 & 3.64 \\
\hline 0.05 & 0.26 & 1.94 & 20.64 & 19.23 & 6.83 & 0.14 & 0.65 & 2.10 & 20.36 & 19.82 & 2.65 \\
\hline 0.05 & 0.52 & 1.94 & 19.52 & 19.47 & 0.26 & 0.26 & 0.0068 & 1.62 & 25.96 & 25.19 & 2.97 \\
\hline 0.05 & 0.52 & 2.10 & 17.66 & 17.99 & 1.87 & 0.26 & 0.0068 & 1.78 & 22.82 & 22.92 & 0.44 \\
\hline 0.05 & 0.65 & 1.45 & 24.18 & 26.01 & 7.57 & 0.26 & 0.0068 & 1.94 & 20.65 & 21.03 & 1.84 \\
\hline 0.05 & 0.65 & 1.62 & 22.59 & 23.43 & 3.72 & 0.26 & 0.0068 & 2.10 & 19.26 & 19.43 & 0.88 \\
\hline 0.05 & 0.65 & 1.78 & 22.22 & 21.32 & 4.05 & 0.26 & 0.26 & 1.45 & 30.68 & 29.89 & 2.58 \\
\hline 0.05 & 0.65 & 1.94 & 20.53 & 19.56 & 4.72 & 0.26 & 0.26 & 1.62 & 26.60 & 26.93 & 1.24 \\
\hline 0.14 & 0.0068 & 1.45 & 27.13 & 26.25 & 3.24 & 0.26 & 0.26 & 2.10 & 21.31 & 20.76 & 2.58 \\
\hline 0.14 & 0.0068 & 1.62 & 23.05 & 23.65 & 2.60 & 0.26 & 0.65 & 1.62 & 27.33 & 27.39 & 0.22 \\
\hline 0.14 & 0.0068 & 1.78 & 22.05 & 21.52 & 2.40 & 0.26 & 0.65 & 1.78 & 25.41 & 24.92 & 1.93 \\
\hline 0.14 & 0.0068 & 1.94 & 19.70 & 19.74 & 0.20 & 0.26 & 0.65 & 1.94 & 23.02 & 22.86 & 0.70 \\
\hline
\end{tabular}

$A-p_{m}$ : actually measured $p_{m} ; C-p_{m}$ : calculated $p_{m} ; R$ : relative error (\%).

TABLE 9: Comparison of peak pressure in two different media of water bottom (unit: MPa).

\begin{tabular}{|c|c|c|c|c|c|}
\hline \multirow[b]{2}{*}{$\bar{H}$} & \multirow[b]{2}{*}{$\bar{h}$} & \multirow[b]{2}{*}{$\bar{L}$} & \multirow[b]{2}{*}{$\bar{r}$} & \multicolumn{2}{|c|}{$p_{m}$} \\
\hline & & & & $\begin{array}{l}\text { Water bottom of soft } \\
\text { mud } p_{1 m}\end{array}$ & $\begin{array}{l}\text { Water bottom of soft } \\
\text { mud with rocks } p_{2 m}\end{array}$ \\
\hline \multirow{10}{*}{0.053} & \multirow{2}{*}{0.0068} & 1.4522 & 1.4529 & 17.97 & 26.56 \\
\hline & & 1.9408 & 1.9410 & 12.30 & 17.97 \\
\hline & \multirow{4}{*}{0.2579} & 1.4522 & 1.4666 & 18.51 & 24.80 \\
\hline & & 1.6151 & 1.6280 & 16.41 & 22.66 \\
\hline & & 1.7779 & 1.7897 & 16.41 & 21.70 \\
\hline & & 2.1037 & 2.1137 & 14.45 & 20.31 \\
\hline & \multirow{2}{*}{0.5157} & 1.9408 & 1.9952 & 14.45 & 23.83 \\
\hline & & 2.1037 & 2.1540 & 12.50 & 20.31 \\
\hline & \multirow{2}{*}{0.6515} & 1.4522 & 1.5707 & 20.70 & 23.83 \\
\hline & & 1.6151 & 1.7224 & 18.36 & 22.36 \\
\hline \multirow{6}{*}{0.136} & \multirow{3}{*}{0.0068} & 1.4522 & 1.4579 & 20.70 & 27.16 \\
\hline & & 1.9408 & 1.9451 & 12.89 & 19.66 \\
\hline & & 2.1037 & 2.1077 & 14.06 & 17.10 \\
\hline & \multirow{3}{*}{0.6515} & 1.4522 & 1.5410 & 20.69 & 25.78 \\
\hline & & 1.9408 & 2.0081 & 17.97 & 22.27 \\
\hline & & 2.1037 & 2.1659 & 16.41 & 20.31 \\
\hline \multirow{8}{*}{0.258} & \multirow{3}{*}{0.0068} & 1.4522 & 1.4738 & 25.78 & 29.30 \\
\hline & & 1.6151 & 1.6345 & 17.97 & 28.91 \\
\hline & & 2.1037 & 2.1186 & 15.23 & 21.88 \\
\hline & \multirow{5}{*}{0.2579} & 1.4522 & 1.4522 & 20.31 & 30.66 \\
\hline & & 1.6151 & 1.6151 & 22.31 & 26.56 \\
\hline & & 1.6151 & 1.6151 & 22.79 & 27.34 \\
\hline & & 1.9408 & 1.9408 & 19.53 & 23.05 \\
\hline & & 2.1037 & 2.1037 & 15.63 & 20.51 \\
\hline
\end{tabular}




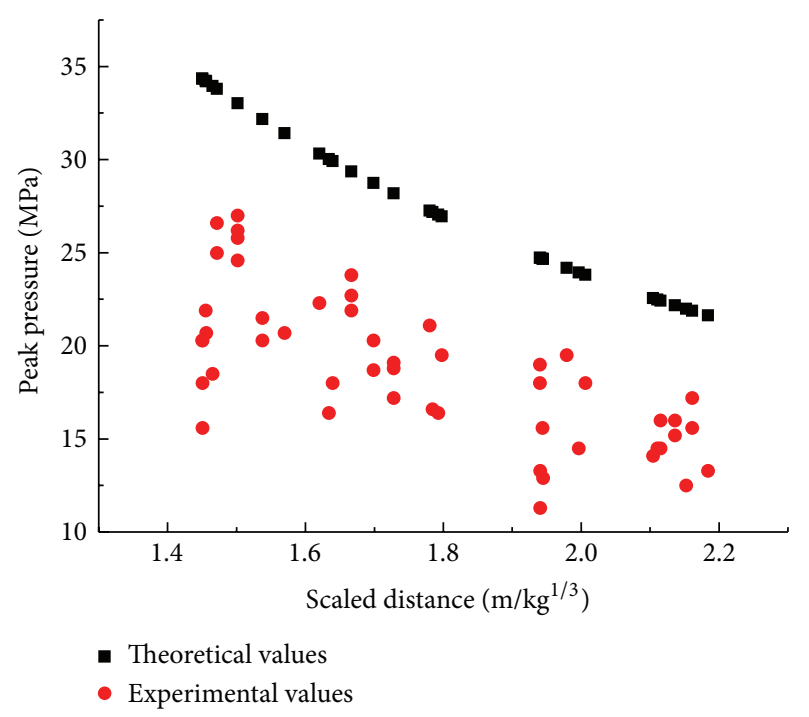

(a) Water bottom of soft mud

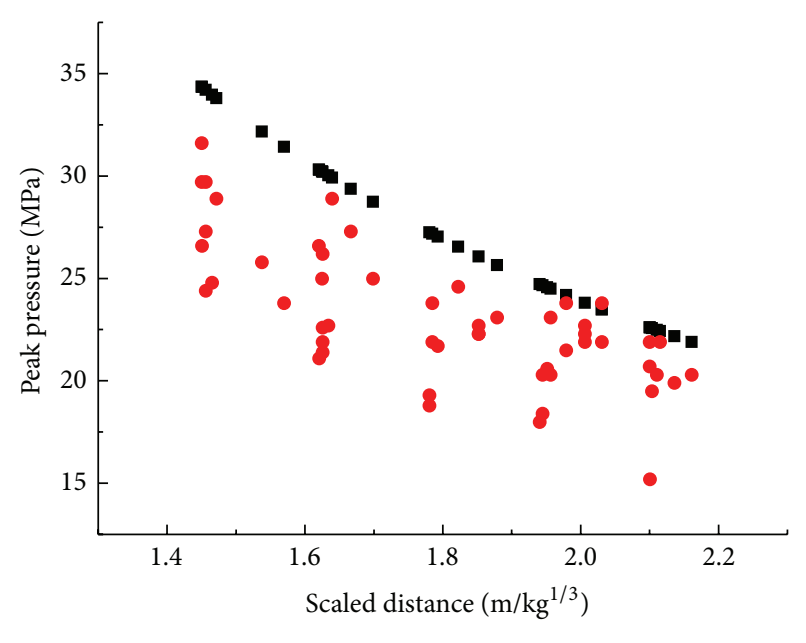

- Theoretical values

- Experimental values

(b) Water bottom of mud with rocks

FIGURE 5: Change curve of peak pressure of shock wave's change with proportional distance in different media of water bottom.

Statistical results of Table 9 display that peak pressure at the water bottom of soft mud with rocks is greater than that of soft mud, indicating that the harder the medium at the water bottom is, the greater the explosion load of charging in quasi-shallow water is, and the better the destruction of the explosion can be improved. Based on data in Table 9 and numerous experimental data, relationship between $p_{1 m}$ and $p_{2 m}$ can be obtained by linear regression:

$$
p_{2 m}=1.33 p_{1 m} .
$$

Under the same experiment conditions in quasi-shallow water explosion, peak pressure of the shock wave in the water bottom of mud with rocks is 1.33 times that of the water bottom of soft mud,

$$
\bar{r}=\sqrt{(\bar{H}-\bar{h})^{2}+\bar{L}^{2}}
$$

Fitting curve of experimentally measured $\ln p_{m}$ of different water bottom and $\ln \bar{r}$ can be shown in Figure 6 and responding functional relations are

with water bottom of soft mud

$$
\ln p_{m 1}=3.42-0.99 \ln \bar{r},
$$

with water bottom of soft mud with rocks

$$
\ln p_{m 2}=3.63-0.85 \ln \bar{r} .
$$

And exponential forms are

$$
\begin{aligned}
& p_{m 1}=30.55 \bar{r}^{-0.99}(\mathrm{MPa}), \\
& p_{m 2}=37.84 \bar{r}^{-0.85}(\mathrm{MPa}) .
\end{aligned}
$$

Analysis on experimentally measured values and results of regression reveals the following:

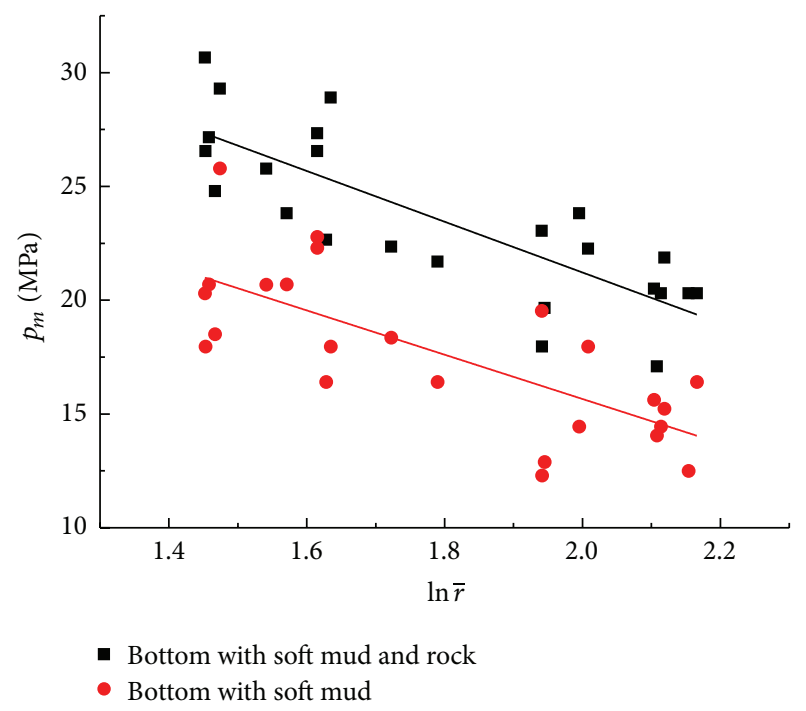

FIGURE 6: Fitting curve of peak pressure of shock wave with two media of water bottom.

(1) In (27) and (29) of two different water bottoms, peak pressure of shock wave increases as the increase of explosion height and measuring height, and the influence of explosion height is greater than that of measuring height; besides, peak pressure of measuring points decreases with the increase of horizontal distance, and the influence is greater than explosion height and measuring height; meanwhile, peak pressure at measuring points in water is smaller than that at the same position in the infinite water explosion.

(2) Comparison of (33) and (34) indicates that, in the explosion of quasi-shallow water at the water bottom of soft mud, peak pressure of the shock wave 


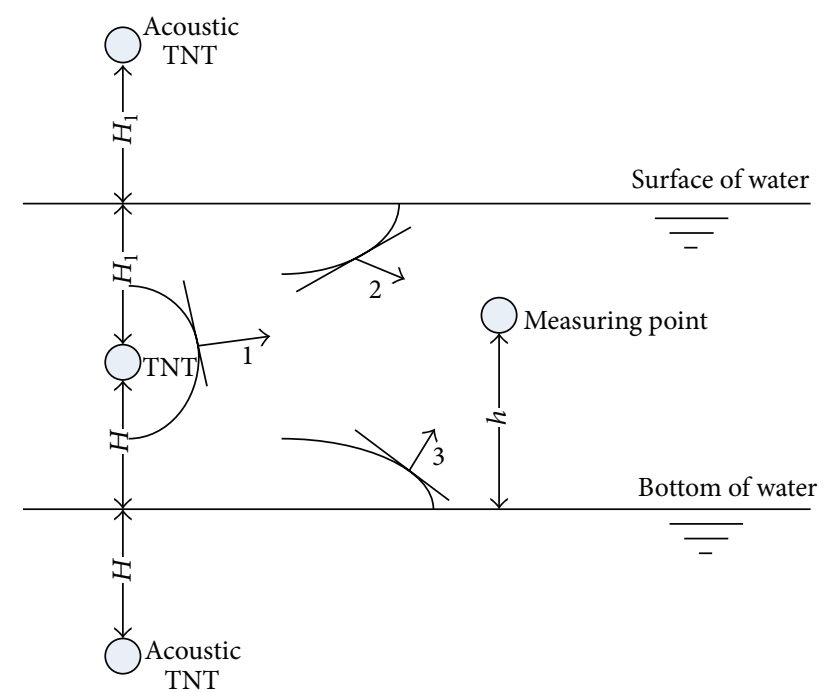

FIGURE 7: Diagram of shock wave pressure in water at measuring points.

attenuates faster than that in the water bottom of mud with rocks, and amplitude of peak pressure at the water bottom of soft mud is smaller than that of the water bottom of soft mud with rocks.

\section{Discussions}

In quasi-shallow water explosion, part to the energy is consumed on crushing or throwing of a medium in the water bottom. Some energy transforms into vibration energy and transmits in the form of seismic wave propagation at the water bottom, and part of energy transmits in the form of the underwater shock wave. And distribution of the above three categories is difficult to study both theoretically and experimentally. Disturbance generated in water spreads between free surface and bottom surface to the water, reflects at the interface, interacts, and causes complex wave system. Therefore, the combined effect of free surface and bottom surface should be taken into consideration when studying explosion in quasi-shallow water [17]. Underwater shock wave generated by the charge explosion in water spreads outward spherically and directly to measuring points, as is shown in Figure 7(1). When the shock wave spreads to the free water surface, it reflects and, similar to the shock wave of the mirrored explosion in air, reflected wave continues to spread in water, as is shown in Figure 7(2). As influence of free surface, shock wave of the underwater explosion acts as the water bottom to make it transform quickly and compacted, generating the reflected wave to spread as Figure 7(3). With different relative position of charging and measuring points, pressure at the points may be unlikely. Peak pressure of measuring points is the result of joint action of Figures $7(1)$, (2), and (3). However, different relative position may cause unlikely influences of the three.

Numerous documents reveal that peak pressure of the underwater shock wave exponentially attenuates with an increase of its distance with charging distance, thus, in influencing factors of peak pressure of underwater shock wave, distance between measuring points and charging has the greatest influence. Moreover, explosion height of charging also directly impacts peak pressure of the shock wave. Measuring height of measuring points and explosion height of charging as well as distance between measuring points and the charging has certain relationships, and measuring height can be determined by distance between measuring pints and charging, horizontal distance, and explosion height, so measuring heights also significantly affect peak pressure of the shock wave at measuring points.

By analyzing the experimental data, it is obtained that water depth does not significantly affect peak pressure of shock wave, but it cannot be concluded that water depth has relatively smaller impact on underwater shock wave. Water depth directly impacts distance between charging water surface and water bottom. According to boundary effect of free water surface and water bottom on shock wave, water depth and medium at the water bottom affect pressure time history curve of the shock wave at measuring points. At different measuring points, influence of water surface and underwater reflected shock wave also varies. There is also the document showing similar conclusions [18], proposing that water depth has relatively less impact on peak pressure. And within a certain proportional distance, when measuring height is certain, increase of explosion height will increase pressure of measuring points with the same proportional distance.

According to the literature [13], if charging explosion energy cannot be absorbed by the water bottom, charging explosion in the water bottom is equivalent to that of a double cartridge of infinite water. Assuming that energy is concentrated in water at the bottom of the water, as an approximation, peak pressure of the double cartridges at any distance increases to $21 / 3=1.26$ times, duration and impulse will also increase, and energy flux density doubles. However, the water bottom cannot be rigid interface and will inevitably absorb part of explosion energy; different media of the water bottom may consume different explosion energy. Moreover, water bottom and reflected wave of water surface have impact; thus, intensity of the shock wave in water may be less than approximation of the double cartridges. Underwater reflected wave has the complex effect on transmission of the underwater shock wave, directly influencing peak pressure of water impact wave. And it is relevant to not only the angle of incidence and angle of reflection, but also properties of underwater media. According to the experimental studies during recent years, when the charging explodes near the water bottom of soft mud, what is, firstly, reflected is not the shock wave (or compression wave); it is reflected sparse wave. With compaction of a medium in the water bottom, reflected compression wave will be reflected later [19]. Effect of the water bottom on underwater shock wave is contrary to that of free surface. When the reflection is generated in underwater sediment layer, rock layer, or surface of other huge obstacles, interaction of reflected wave and waves spreading in water will affect peak pressure of the shock wave. Experiment data of the explosion in different media of the water bottom 
indicate that peak pressure of the shock wave in the water bottom of mud with rocks is about 1.33 times that of the water bottom with soft mud. Given that water bottom is the absolutely rigid boundary, it may be obtained that underwater shock wave reflects the compression wave, with the same intensity and incident. But it is not in real conditions; as media of water bottom and reflection coefficients are different, peak pressure at measuring points will also vary. Hence, influence of a medium at the water bottom on peak pressure of the underwater shock wave cannot be ignored. Peak pressure of the shock wave in the water bottom of soft mud decreases more quickly than that of the water bottom of soft mud with rocks, demonstrating that water bottom of soft mud absorbs more explosion energy. The higher the rigidity of a medium of the water bottom is, the more the peak pressure of the underwater shock wave will increase.

\section{Conclusions}

By establishing regression model and analysis method, factors impacting peak pressure of the shock wave in a quasishallow water explosion are discussed in detail. Regression analysis and corresponding experiment have been carried out on the model of experiment data, and, on the model, following conclusions can be drawn:

(1) Explosion height, measuring height, and water depth as well as horizontal distance all influence peak pressure of the shock wave at measuring points. Horizontal distance from charging center to measuring points has the most significant impact on peak pressure of incident wave front, followed by explosion height, and then comes measuring height, and water depth's influence is not significant within the range of geometric parameters of these experiments.

(2) Data calculated by the formula of calculating models are more accurate and calculating results have relatively high accuracy within the formula scope.

(3) Explosion experiment of two different media of the water bottom reveals that the media significantly impact peak values of the shock wave. And, under the same experiment conditions, peak pressure through the medium of soft mud with rocks is 1.33 times that of the soft mud water bottom.

(4) Numerous factors influence peak pressure of the underwater shock wave, and plenty of experiment analysis is required for thoroughly exploring transmission law of the underwater shock wave. Due to financial constraints and experimental conditions, certain limitations exist in resulting experiment data in the present paper.

\section{Conflict of Interests}

The authors declare that there is no conflict of interests regarding the publication of this paper.

\section{Acknowledgments}

This work was supported by a grant from National Defense Program, Grant no. BY209J033. Their contribution to bringing this project to fruition is much appreciated. And high tribute shall be paid to Liang Ting, who has provided insightful comments on editing and translating for this thesis.

\section{References}

[1] W.-B. Gu, Experimental Investigation and Numerical Simulation Studies of Propagating Characters of Exploding Shock Waves in Shallow-Layer Water, PLA University of Science and Technology, 2000.

[2] R. H. Cole, Underwater Explosions, Princeton University Press, Princeton, NJ, USA, 1961.

[3] J. P. Slifko, "Pressure-pulse characteristics of deep explosion as functions of depth and range," Tech. Rep. AD-661804, 1967.

[4] K. Hanasaki and I. Ito, "Determination of the underwater shock pressure by measuring the particle velocity on the water surface," Science and Technology of Energetic Materials, vol. 35, no. 6, pp. 275-283, 1974.

[5] K. Takayama and O. Onodera, "Holographic interferometric study on propagating and focusing of underwater shock waves by micro-explosions," in Optical Methods in Dynamics of Fluids and Solids, pp. 209-216, Springer, Berlin, Germany, 1985.

[6] M. M. Swisdak Jr., "Explosion effects and properties: part II. Explosion effects in water," Tech. Rep. AD-A056694, 1978.

[7] A. V. Adushkin, V. N. Burchik, A. I. Goncharov, V. I. Kulikov, B. D. Khristoforov, and V. I. Tsykanovskii, "Seismic, hydroacoustic, and acoustic action of underwater explosions," Combustion, Explosion and Shock Waves, vol. 40, no. 6, pp. 707-713, 2004.

[8] V. V. Ostapenko, "Stable shock waves in two-layer 'shallow water"' Journal of Applied Mathematics and Mechanics, vol. 65, no. 1, pp. 89-108, 2001.

[9] L. Zhou, Z. Xie, and X. Wei, "Comparison of underwater shock wave attenuation of a new insensitive high explosive with different explosives," Combustion, Explosion and Shock Waves, vol. 47, no. 6, pp. 721-726, 2011.

[10] S. K. Kwang, "Comment on Underwater Explosion (UWE) analysis of the ROKS cheonan incident by S. G. Kim and Y. Kitterman," Pure and Applied Geophysics, vol. 170, no. 3, pp. 473478, 2013.

[11] Zamyshlysaev, "Pressure fields during underwater explosion in a free fluid," Dynamic Loads in Underwater Explosion, vol. 7, no. 2, pp. 86-120, 1973.

[12] P.-X. Zhang, W.-B. Gu, and X.-S. Ye, "Discussion on calculation method of peak pressure subjected to shallow-layer water explosion," Journal of PLA University of Science and Technology, vol. 3, no. 1, 2002.

[13] X.-Q. Liang, Technology of Underwater Blasting, Chemical Industry Press, Beijing, China, 2013.

[14] X.-S. Ye, Basis of Explosion, Engineering Institute of Corps of Engineers, Nanjing, China, 1996.

[15] H. Li, Digital Signal Processing and Its MATLAB Implementation, China Machine Press, Beijing, China, 2011.

[16] T. Saito, M. Marumoto, H. Yamashita et al., "Experimental and numerical studies of underwater shock wave attenuation," Shock Waves, vol. 13, no. 2, pp. 139-148, 2003.

[17] Ao. Li Er Ke, Explosion Physics, Science Press, Beijing, China, 2011. 
[18] W.-B. Gu, X.-S. Ye, W.-H. Liu, and Q. Liu, "Peak pressure investigation of exploding wave influenced by interfaces in shallow-layer water," Journal of PLA University of Science and Technology, vol. 2, no. 5, 2001.

[19] X.-S. Ye, Basis of Dynamics Explosion, Engineering Institute of Corps of Engineers, PLA University of Science and Technology, Nanjing, China, 2004. 


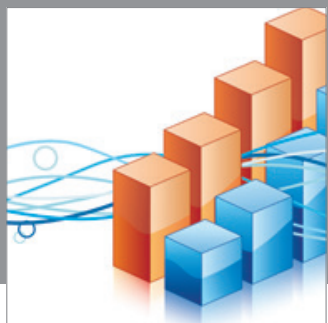

Advances in

Operations Research

mansans

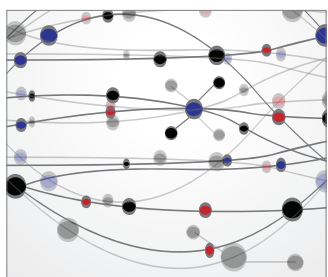

The Scientific World Journal
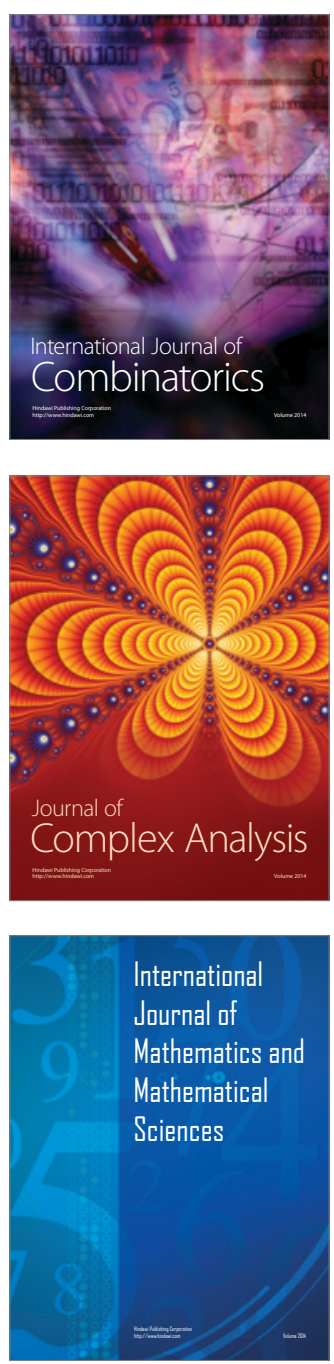
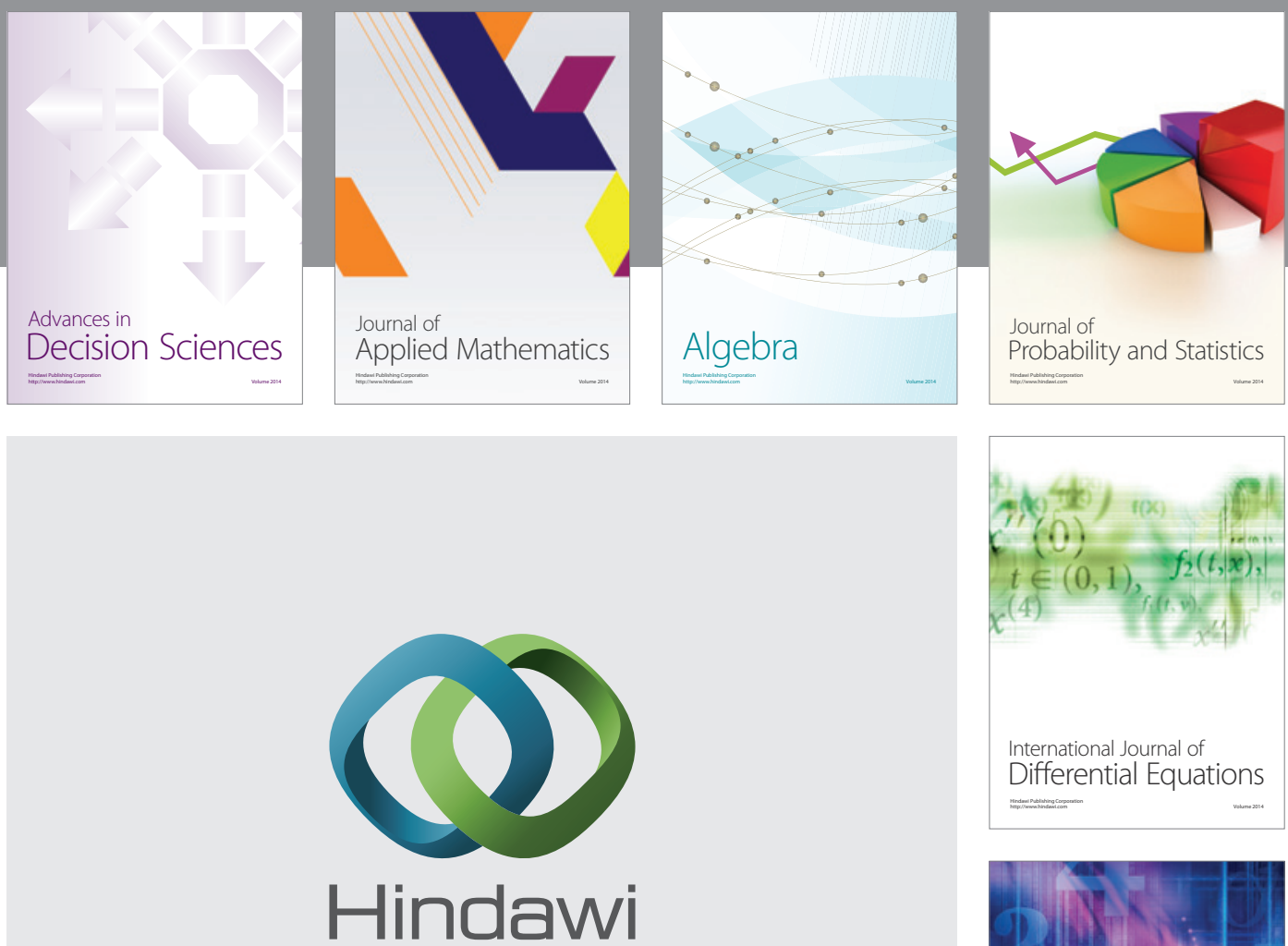

Submit your manuscripts at http://www.hindawi.com
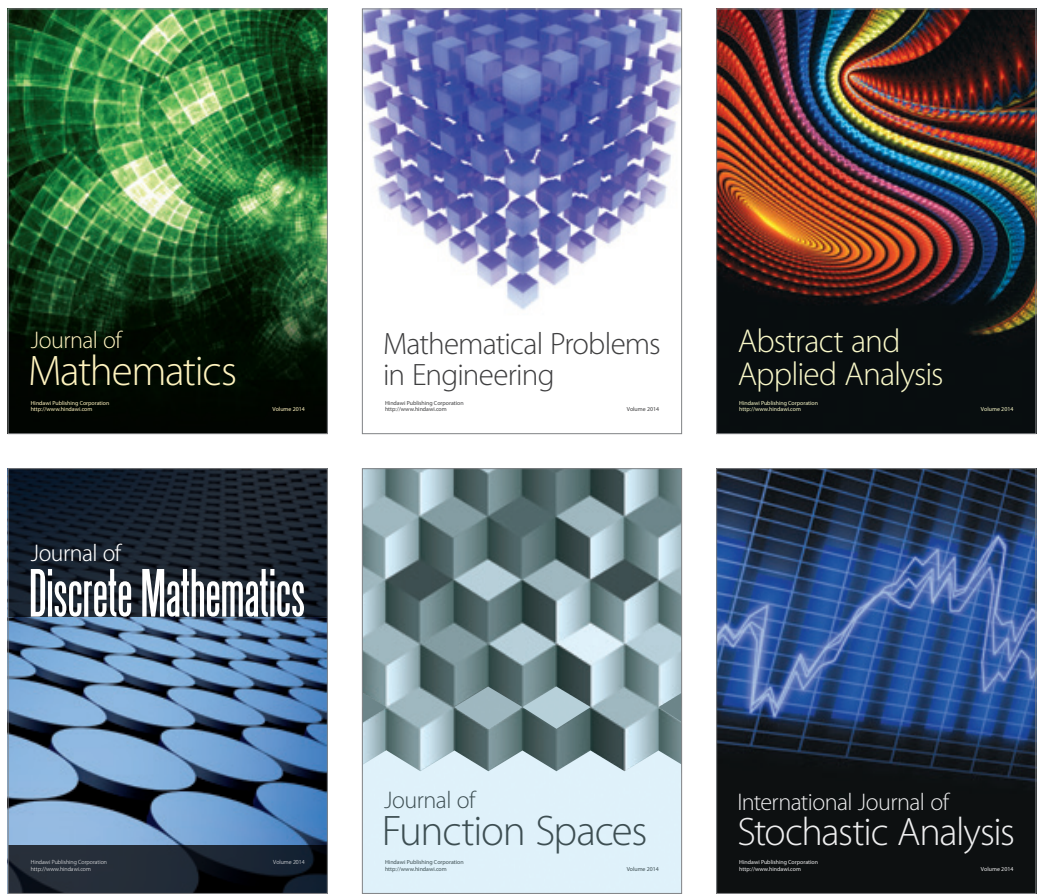

Journal of

Function Spaces

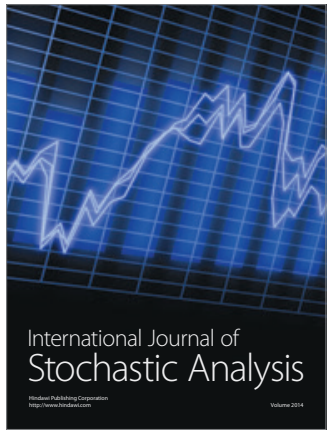

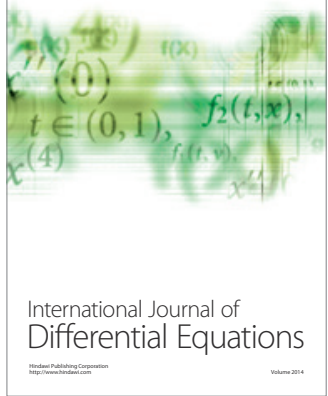
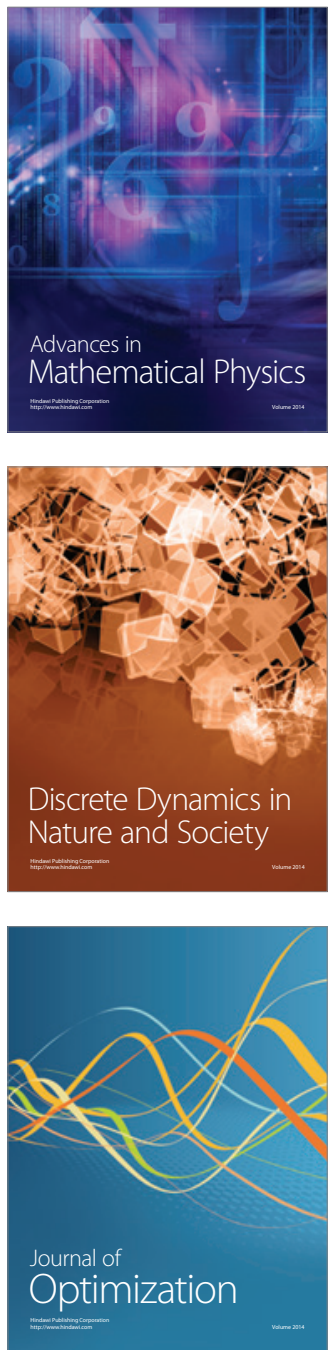